\title{
COMPARING CORRESPONDING DIHEDRAL ANGLES ON CLASSICAL GEOMETRIC SIMPLICES*
}

\author{
THOMAS KWOK-KEUNG AU ${ }^{\dagger}$, FENG LUO $¥$, AND RICHARD STONG §
}

\begin{abstract}
In this article, we prove a theorem comparing the dihedral angles of simplexes in the hyperbolic, spherical and Euclidean geometries.
\end{abstract}

AMS subject classifications. 51K10, 53A99

1. Introduction. It is well known that given any spherical (or hyperbolic) triangle in dimension 2, one can decrease (or increase) its inner angles to obtain an Euclidean triangle. The goal of this paper is to establish this general fact for all dimensions. This study was motivated by the study of the volume of convex polytopes in classical geometry in terms of dihedral angles. Interesting related topics can also be found in [Luo2, Luo3].

By a space of classical geometry, we mean the $n$-sphere $\mathbb{S}^{n}$, the Euclidean $n$ space $\mathbb{E}^{n}$, or the hyperbolic $n$-space $\mathbb{H}^{n}$. For simplicity, they will be collectively denoted by $\mathbb{K}^{n}$. A classical geometric $n$-simplex $\mathcal{Z}$ in $\mathbb{K}^{n}$ or simply a $\mathbb{K}^{n}$-simplex is the geodesic convex hull of $(n+1)$ points $z_{1}, z_{2}, \ldots, z_{n+1}$ in $\mathbb{K}^{n}$ so that these points are not lying in any $(n-1)$-dimensional totally geodesic submanifold. These $n+1$ points are called the vertices of the simplex $\mathcal{Z}$. As a convention, we always consider simplexes with vertex labelled. That is, the simplex $\mathcal{Z}$ is represented by the $(n+1)$-tuple $\left(z_{1}, z_{2}, \ldots, z_{n+1}\right) \in\left(\mathbb{K}^{n}\right)^{n+1}$, where $z_{i}$ denotes the $i$-th vertex. In addition, two simplexes are equivalent if there is a $\mathbb{K}^{n}$-isometry taking such an $(n+1)$ tuple to another.

We will compare the dihedral angles of simplexes in classical geometries. Let $\mathcal{Z}=\left(z_{1}, z_{2}, \ldots, z_{n+1}\right)$ be a $\mathbb{K}^{n}$-simplex. We denote the codimension- 1 face opposite to the $i$-th vertex $z_{i}$ by $F_{i}(\mathcal{Z})=\left(z_{1}, \ldots, \ldots, \varkappa_{i}, \ldots, z_{n+1}\right) \in\left(\mathbb{K}^{n}\right)^{n}$. Then the dihedral angle $\zeta_{i j}$, for $i \neq j$, is the angle between the faces $F_{i}(\mathcal{Z})$ and $F_{j}(\mathcal{Z})$. Let $\mathcal{S}$ and $\mathcal{T}$ be two $\mathbb{K}^{n}$-simplexes (not necessarily the same $\mathbb{K}^{n}$ ) of dihedral angles $\sigma_{i j}$ and $\tau_{i j}$ respectively. It is said that $\mathcal{S} \preceq \mathcal{T}$ if and only if $\sigma_{i j} \leq \tau_{i j}$ for every $i, j$. If in addition, there is a pair of $i \neq j$ such that $\sigma_{i j}<\tau_{i j}$, then $\mathcal{S} \prec \mathcal{T}$.

In this article, we are going to demonstrate a theorem which may be roughly abbreviated by $\mathbb{H}^{n} \prec \mathbb{E}^{n} \prec \mathbb{S}^{n}$.

TheOrem (COMPARISOn of SimpleXes). There is a natural partial order on $n$-simplexes in these spaces of classical geometry according to dihedral angles. More precisely,

M1 For every $\mathbb{S}^{n}$-simplex $\mathcal{S}$, there is an $\mathbb{E}^{n}$-simplex $\mathcal{E}$ such that $\mathcal{E} \prec \mathcal{S}$.

M2 For every $\mathbb{H}^{n}$-simplex $\mathcal{H}$, there is an $\mathbb{E}^{n}$-simplex $\mathcal{E}$ such that $\mathcal{H} \prec \mathcal{E}$.

M3 For every $\mathbb{E}^{n}$-simplex $\mathcal{E}$, there is an $\mathbb{S}^{n}$-simplex $\mathcal{S}$ and an $\mathbb{H}^{n}$-simplex $\mathcal{H}$ such that $\mathcal{H} \prec \mathcal{E} \prec \mathcal{S}$.

\footnotetext{
${ }^{*}$ Received June 7, 2006; accepted for publication November 15, 2007.

$\dagger$ Department of Mathematics, The Chinese University of Hong Kong, Shatin, Hong Kong (thomasau@cuhk.edu.hk).

${ }^{\ddagger}$ Center of Mathematical Sciences, Zhejiang University, Hangzhou, China; Department of Mathematics, Rutgers University, Piscataway, NJ08854, USA (fluo@math.rutgers.edu).

$\S$ Department of Mathematics, Rice University, Houston, TX77005, USA (stong@math.rice.edu).
} 
M4 if $\mathcal{E}_{1}, \mathcal{E}_{2}$ are $\mathbb{E}^{n}$-simplexes such that $\mathcal{E}_{1} \preceq \mathcal{E}_{2}$, then $\mathcal{E}_{1}$ and $\mathcal{E}_{2}$ have exactly the same corresponding dihedral angles.

Remark. The statement M4 above might have been known for long. To our knowledge, it was posted as a problem in [Riv2] by Rivin and a composite solution was given later in [LL]. It had also been proved by Richard Stong independently. In addition, the theorem is trivial for $n=2$.

The statements M3 and M4 are proved in $\S 2$ by considering suitable variations of Gram matrices. The proof reflects variational properties in addition to simply angle comparison. In a certain sense, Euclidean Gram matrices lie in the common boundary of spherical and hyperbolic ones. In $\S 3$, we will prove M2 using geometric comparison. A given $\mathbb{H}^{n}$-simplex in the disk model always determines an inscribed $(n-1)$-sphere. This sphere in turns is inscribed by the desired $\mathbb{E}^{n}$-simplex and the dihedral angle comparison follows naturally from Gauss-Bonnet Theorem. Both the above methods do not work for proving M1. On the one hand, the method of varying Gram matrices fails because we do not have control of the signs of cofactors. On the other hand, unlike the case of an $\mathbb{H}^{n}$-simplex, tangent hyperplanes of a $\mathbb{S}^{n}$-simplex do not necessarily bound a compact region. In order to geometrically construct the desired $\mathbb{E}^{n}$-simplex, it requires more subtle technique. The idea is to "extend" or "enlarge" the dual of the given $\mathbb{S}^{n}$-simplex. Then take the Euclidean dual of the "extended" simplex and perturb a little bit if necessary. The details will be discussed in $\S 4$.

2. Gram Matrices. The Gram matrix $G=G(\mathcal{Z})$ of a $\mathbb{K}^{n}$-simplex $\mathcal{Z}$ is an $(n+1) \times(n+1)$ matrix with entries $-\cos \zeta_{i j}$, where $\zeta_{i j}$ is the dihedral angles of $\mathcal{Z}$ with the convention $\zeta_{i i}=\pi$. It is clearly symmetric and has diagonal entries equal to 1 . Since the function $-\cos (\cdot)$ is monotonic increasing on $(0, \pi)$, it is also natural to say that two Gram matrices $\left(a_{i j}\right) \preceq\left(b_{i j}\right)$ if their corresponding entries $a_{i j} \leq b_{i j}$ for all $i, j$.

REMARK. This matrix is sometimes called angle Gram matrix to distinguish it from the so-called length Gram matrix.

First, let us recall a clarification of the relation between Gram matrices and classical geometric simplexes. It was first proved in [Mil] and it can also be found in [AVS] and [Luo1]. There are also interesting related works, [Dia, RH, Riv1, Riv3].

TheOREM 1. Let $A$ be an $(n+1) \times(n+1)$ real symmetric matrix with diagonal entries equal 1 and let $c_{i j}$ be the $(i, j)^{\text {th }}$ cofactor of $A$.

1. A is the Gram matrix of an $\mathbb{S}^{n}$-simplex if and only if $A$ is positive definite.

2. A is the Gram matrix of an $\mathbb{E}^{n}$-simplex if and only if $\operatorname{det}(A)=0$, all principal $n \times n$ submatrices of $A$ are positive definite, and all $c_{i j}>0$.

3. $A$ is the Gram matrix of an $\mathbb{H}^{n}$-simplex if and only if $\operatorname{det}(A)<0$, all principal $n \times n$ submatrices of $A$ are positive definite, and all $c_{i j}>0$.

For simplicity, we may refer to the above cases of Gram matrices as spherical, Euclidean, or hyperbolic Gram matrices.

Using continuous variation of Gram matrices, we are able to show that an Euclidean simplex sits between a hyperbolic and a spherical ones.

TheOrem M3. For any Euclidean $n$-simplex $\mathcal{E}$, there is a hyperbolic $n$-simplex $\mathcal{H}$ and a spherical $n$-simplex $\mathcal{S}$ such that $\mathcal{H} \prec \mathcal{E} \prec \mathcal{S}$. 
Proof. Let $\mathcal{E}$ be an $\mathbb{E}^{n}$-simplex and $G=\left(g_{i j}\right)$ be its corresponding Gram matrix with cofactors $c_{i j}$. In other words, by Theorem $1, G$ satisfies the following three conditions, $\operatorname{det}(G)=0 ; c_{i j}>0$ for all $i, j$; and all principle $n \times n$ submatrices of $G$ are positive definite.

Let $P=\left(p_{i j}\right)$ be the $(n+1) \times(n+1)$ matrix in which every diagonal entry is 1 and $p_{i j} \equiv-1$ for all $i \neq j$. Let $A(t)=\left(a_{i j}(t)\right)$ be the path in the space of $(n+1) \times(n+1)$ symmetric matrices defined by,

$$
A(t)=(1-t) G+t P \quad t \in[0,1] .
$$

It is clear that the eigenvalues of the principal $n \times n$ matrices of $A(t)$ and the cofactors $c_{i j}(t)$ of $A(t)$ depend continuously on the entries of $A(t)$ and hence in $t$. Thus, for sufficiently small $t>0$, the principal $n \times n$ matrices remain positive definite and $c_{i j}(t)>0$. Moreover,

$$
\frac{d}{d t}[\operatorname{det} A(t)]=\sum_{i, j=1}^{n+1} c_{i j}(t) a_{i j}^{\prime}(t)=\sum_{i \neq j} c_{i j}(t)\left(-1-g_{i j}\right) .
$$

Since $c_{i j}(0)>0$, we have $\frac{d}{d t}[\operatorname{det} A(t)]_{t=0}<0$ and $\operatorname{det} A(t)<0$ for sufficiently small $t>0$. Thus, again by Theorem $1, A(t)$ corresponds to the Gram matrix of a $\mathbb{H}^{n}$ simplex $\mathcal{H}$. Clearly, $a_{i j}(t)<g_{i j}$ for $i \neq j$.

To obtain an $\mathbb{S}^{n}$-simplex $\mathcal{S}$, one simply takes another matrix $P$ which has all entries $p_{i j} \equiv 1$ for all $i, j$. This clearly produces $a_{i j}(t)>g_{i j}$ for $i \neq j$. The argument is exactly the same as above with the only difference that $\operatorname{det}(A(t))>0$. As a result, $A(t)$ corresponds to the Gram matrix of a $\mathbb{S}^{n}$-simplex $\mathcal{S}$. It is then concluded that $\mathcal{H} \prec \mathcal{E} \prec \mathcal{S}$. $\square$

Remark. From the proof, we actually have $\mathcal{S}$ and $\mathcal{H}$ which have dihedral angles arbitrarily close to those of $\mathcal{E}$.

The Gram matrices also provides another proof for the "rigidity" of Euclidean simplexes given by Stong.

THEOREM M4. If $\mathcal{E}_{1}$ and $\mathcal{E}_{2}$ are two Euclidean $n$-simplexes such that $\mathcal{E}_{1} \preceq \mathcal{E}_{2}$, then they are similar.

Proof. Let $\mathcal{E}_{1}$ and $\mathcal{E}_{2}$ be two Euclidean $n$-simplexes such that $\mathcal{E}_{1} \preceq \mathcal{E}_{2}$. Furthermore, let $G_{1}$ and $G_{2}$ be their corresponding Gram matrices and $A(t)=(1-t) G_{1}+t G_{2}$, $t \in[0,1]$ be a path in symmetric matrices joining the two Gram matrices. We also denote the cofactors of $A(t)$ by $c_{i j}(t)$.

By Theorem 1, all principal $n \times n$ submatrices of $G_{1}$ and $G_{2}$ are positive definite and $\operatorname{det}\left(G_{1}\right)=0=\operatorname{det}\left(G_{2}\right)$. Thus, both $G_{1}$ and $G_{2}$ are semi-positive definite. As a consequence, $A(t)$ is semi-positive definite for all $t$. In particular, $\operatorname{det}(A(t)) \geq 0$ for all $t \in[0,1]$. Let $f(t)=\operatorname{det}(A(t))$. It is obvious that

$$
f^{\prime}(t)=\frac{d}{d t}(\operatorname{det}(A(t)))=\sum_{i \neq j}\left(\cos \alpha_{i j}-\cos \beta_{i j}\right) c_{i j}(t),
$$

where $\alpha_{i j}$ and $\beta_{i j}$ are the dihedral angles of the simplexes $\mathcal{E}_{1}$ and $\mathcal{E}_{2}$ respectively. Note that for all $i, j, \alpha_{i j} \leq \beta_{i j}$, thus $\cos \alpha_{i j} \geq \cos \beta_{i j}$. Suppose there is a pair of corresponding dihedral angles $\alpha_{p q}<\beta_{p q}$, then we will derive a contradiction. 
Since $G_{1}$ and $G_{2}$ are Euclidean Gram matrices, for all $i, j$, we have $c_{i j}(0)>0$ and $c_{i j}(1)>0$. As a consequence,

$$
f^{\prime}(1) \geq\left(\cos \alpha_{p q}-\cos \beta_{p q}\right) c_{p q}(1)>0 .
$$

Together with the fact that $f(1)=0$, there is a small $\varepsilon>0$ such that $f(t)<0$ for $t \in(1-\varepsilon, 1)$. This contradicts that $f(t) \geq 0$. Hence, for all $i, j$, one must have $\alpha_{i j}=\beta_{i j}$.

3. Gauss-Bonnet. To show that a hyperbolic simplex is dominated by an Euclidean one, it only requires a simple geometric construction and an angle comparison based on the Gauss-Bonnet Theorem.

THEOREM M2. For every hyperbolic $n$-simplex $\mathcal{H}$, there is an Euclidean $n$-simplex $\mathcal{E}$ such that $\mathcal{H} \prec \mathcal{E}$.

Let $\mathcal{H}$ be a $\mathbb{H}^{n}$-simplex in the Poincaré disc model $\mathbb{D}^{n}$ of the hyperbolic space. Let $S \subset \mathbb{D}^{n}$ be an inscribed hyperbolic $(n-1)$-sphere of $\mathcal{H}$. Without loss of generality, by a hyperbolic isometry, one may assume that the in-center of $\mathcal{H}$ is the origin and so $S$ is an Euclidean sphere with center at the origin.

Let $u_{1}, \ldots, u_{n+1} \in \mathbb{D}^{n}$ be the points of tangency of $S$ to the faces of $\mathcal{H}$. They are also considered as Euclidean vectors from the origin. Let us first give an algebraic description of the geometry of the vectors.

LEMMA 2.

1. Any $n$ vectors among $\left\{u_{1}, \ldots, u_{n+1}\right\}$ are linearly independent.

2. The system of linear equations $\sum_{i=1}^{n+1} x_{i} u_{i}=0$ has only a 1-dimensional solution space of the form $\left(x_{1}, \ldots, x_{n+1}\right)$ where $x_{i} x_{j}>0$ for all $i, j$. That is, the $x_{i}$ 's are all of the same sign.

Proof. Let $W_{1}, \ldots, W_{n+1} \subset \mathbb{D}^{n}$ be codimension-1 hyperbolic hypersurfaces tangent to $S$ at $u_{1}, \ldots, u_{n+1}$ respectively. That is, each $W_{i}$ contains an $(n-1)$-face of $\mathcal{H}$. Since $\mathcal{H}$ is nondegenerate, the first statement is evident; otherwise, there will be $n$ such faces intersecting in a 1-dimensional geodesic but not a vertex.

Suppose the second statement is not true. By simple Linear Algebra, there is a vector $v$ satisfying $\left\langle v, u_{i}\right\rangle \leq 0$ for all $i=1, \ldots, n+1$. Take a hyperbolic geodesic $L$ from the center of $S$ along the direction $v$. This geodesic makes an angle $\geq \pi / 2$ with each $u_{i}$. If $L$ intersects some $W_{j}$, then $L, u_{j}$, and a geodesic in $W_{j}$ will form a hyperbolic triangle with angle sum $>\pi$. Thus $L$ does not intersect any of $W_{i}$. This contradicts the fact that the hypersurfaces $W_{i}$ bound a compact simplex.

REMARK. Note that in the disk model, the geodesic $L$ from the center is also an Euclidean ray. Thus, the same argument proves an analogue of the second statement in $\mathbb{E}^{n}$.

Proof of M2. Let $P_{1}, \ldots, P_{n+1}$ be the Euclidean codimension-1 hyperplanes in $\mathbb{R}^{n}$ tangent to $S$ at $u_{1}, \ldots, u_{n+1}$ respectively. Then by Lemma 2 , an $\mathbb{E}^{n}$-simplex $\mathcal{E}$ is bounded by $P_{1}, \ldots, P_{n+1}$ with the origin as its in-center.

Since each $P_{i}$ has normal vector $u_{i}$, the dihedral angles $\xi_{i j}$ of $\mathcal{E}$ are given by

$$
\xi_{i j}=\pi-\angle\left(u_{i}, u_{j}\right)=\pi-\arccos \frac{\left\langle u_{i}, u_{j}\right\rangle}{\left\|u_{i}\right\|\left\|u_{j}\right\|} \text {. }
$$


As in the above lemma, we continue to use $W_{1}, \ldots, W_{n+1}$ to denote codimension- 1 hyperbolic hypersurfaces with normals $u_{1}, \ldots, u_{n+1}$; and these $W_{i}$ 's bound the hyperbolic simplex $\mathcal{H}$. Let $\eta_{i j}$ be the hyperbolic dihedral angle between $W_{i}$ and $W_{j}$. Consider the Euclidean 2-plane $P$ through the origin spanned by the vectors $u_{i}$ and $u_{j}$. Then by the construction $P$ is perpendicular to both $P_{i}$ and $P_{j}$. Let $D^{2}$ be the intersection $P \cap \mathbb{D}^{n}$. Then $D^{2}$ is a totally geodesic hyperbolic 2-plane perpendicular to $W_{i}$ and $W_{j}$ (see the figure below).

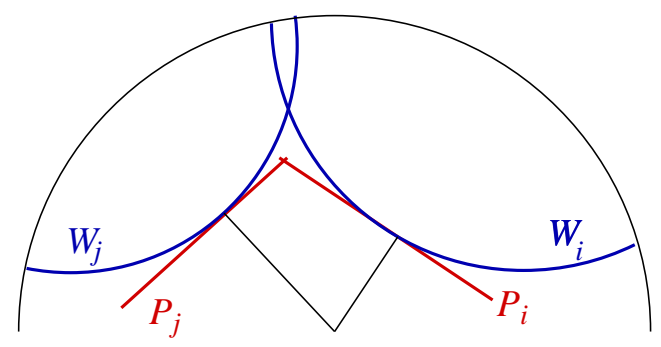

The intersections of $D^{2}$ with $W_{i}$ and $W_{j}$ respectively produce two geodesics $\gamma_{i}$ and $\gamma_{j}$ in $D^{2}$. These two geodesics together with the geodesics $u_{i}$ and $u_{j}$ from the origin form a hyperbolic quadrilateral in $D^{2}$ with inner angles $\pi-\xi_{i j}, \pi / 2, \pi / 2, \eta_{i j}$. By Gauss-Bonnet Theorem, it follows that their sum is less than $\pi$. Thus $\eta_{i j}<\xi_{i j}$ and hence $\mathcal{H} \prec \mathcal{E}$.

4. The Sphere. In this last section, we will deal with spherical simplexes. The following convention will be adopted. Let $\mathbb{S}^{n}$ be the unit sphere in $\mathbb{E}^{n+1} ; \mathbb{E}^{n}=$ $\mathbb{E}^{n} \times\{0\} \subset \mathbb{E}^{n+1}$ and $\mathbb{S}^{n-1}=\mathbb{S}^{n} \cap \mathbb{E}^{n}$.

TheOrem M1. For any spherical n-simplex $\mathcal{S}$ with dihedral angles $\sigma_{i j}$, there is an Euclidean n-simplex $\mathcal{E}$ with dihedral angles $\xi_{i j}$ such that $\xi_{i j}<\sigma_{i j}$ for all $i, j$.

The strategy of the proof goes as follows. Let $\mathcal{S} \subset \mathbb{S}^{n}$ be a spherical simplex with dihedral angles $\sigma_{i j}, i, j=1, \ldots, n+1$. Consider its dual $\mathbb{S}^{n}$-simplex $\mathcal{S}^{*}=$ $\left(v_{1}, \ldots, v_{n+1}\right) \in\left(\mathbb{S}^{n}\right)^{n+1}$. By duality, the spherical distance between the vertices is given by $d_{\mathbb{S}^{n}}\left(v_{i}, v_{j}\right)=\pi-\sigma_{i j}$. We will move the vertices $v_{i}$ 's appropriately to increase the distances $d_{\mathbb{S}^{n}}\left(v_{i}, v_{j}\right)$ until it becomes the so-called spherical dual of an Euclidean $n$-simplex. The Euclidean $n$-simplex will have dihehral angles smaller than $\sigma_{i j}$.

In the rest of the section, for a $k$-ball $B \subset \mathbb{S}^{n}$, by a hemi-sphere in $\partial B$, we refer to a closed $(k-1)$-ball in $\partial B$ of the same radius as $B$.

First, let us recall briefly the dual of an Euclidean $n$-simplex $\mathcal{E}$ in $\mathbb{E}^{n}$. The following is a well-known fact. See, for instance, [Luo1] for a proof.

Lemma 3. Given $n+1$ points $w_{1}, \ldots, w_{n+1} \in \mathbb{S}^{n-1} \subset \mathbb{E}^{n}$, the convex polytope $\mathcal{E}=\left\{x \in \mathbb{E}^{n}:\left\langle x-w_{i}, w_{i}\right\rangle \leq 0\right.$ for all $\left.i\right\}$, which is bounded by the tangent planes to $\mathbb{S}^{n-1}$ at $w_{i}$ 's and contains the origin, is an Euclidean $n$-simplex $\mathcal{E}$ if and only if $\left\{w_{1}, \ldots, w_{n+1}\right\}$ does not lie in any hemi-sphere in $\mathbb{S}^{n-1}$.

We call $\left(w_{1}, \ldots, w_{n+1}\right) \in\left(\mathbb{S}^{n-1}\right)^{n+1}$ the spherical dual of $\mathcal{E}$. Note that the $(i, j)^{\text {th }}$ dihedral angle of $\mathcal{E}$ is $\pi-d_{\mathbb{S}^{n}}\left(w_{i}, w_{j}\right)$.

Second, we need a process of extending the sides of a geodesic triangle on $\mathbb{S}^{n}$.

Lemma 4. Let $T_{0}$ be a spherical triangle of angles $a, b, c$ and corresponding opposite side lengths $x(0), y(0), z(0)$. Let $T_{t}$ be a 1-parameter family of spherical 
triangles obtained by extending the lengths of two sides from $x(0)$ and $y(0)$ to $x(t)$ and $y(t)$ respectively in the same growth rate $x^{\prime}(t)=y^{\prime}(t)=g(t)>0$ while keeping the angle $c$ fixed. If $x(t)+y(t)<\pi$, then the length $z(t)$ of the third side satisfies $z(t)>z(0)$.



Proof. According to the spherical Cosine Law, for each $T_{t}$, we have

$$
\cos z(t)=\cos x(t) \cos y(t)+\sin x(t) \sin y(t) \cos (c) .
$$

Differentiating with respect to $t$ and grouping terms, we have

$$
\begin{aligned}
z^{\prime}(t) \sin z(t)= & x^{\prime}(t) \sin x(t) \cos y(t)+y^{\prime}(t) \cos x(t) \sin y(t) \\
& -x^{\prime}(t) \cos x(t) \sin y(t) \cos (c)-y^{\prime}(t) \sin x(t) \cos y(t) \cos (c) \\
= & g(t)(1-\cos (c)) \sin (x(t)+y(t))>0 .
\end{aligned}
$$

Thus, $z(t)$ keeps increasing as long as the condition $x(t)+y(t)<\pi$ holds.

To begin the proof, consider the dual simplex $\mathcal{S}^{*}=\left(v_{1}, \ldots, v_{n+1}\right) \in\left(\mathbb{S}^{n}\right)^{n+1}$ of the given one $\mathcal{S}$. Let $B_{s} \subset \mathbb{S}^{n}$ be the spherical $n$-ball of the smallest radius containing $\mathcal{S}^{*}$. Without loss of generality, assume its center is located at the south pole $s=(0, \ldots, 0,-1) \in \mathbb{E}^{\mathbf{n}+\mathbf{1}}$. Evidently, its radius $<\pi / 2$ and there are at least two vertices among $v_{i}$ 's lying on the boundary of $B_{s}$. By permutating the vertex labels, we may assume that $v_{1}, \ldots, v_{m} \in \partial B_{s}$ for $2 \leq m \leq n+1$, while $v_{m+i} \in \operatorname{int}\left(B_{s}\right)$ for $i \geq 1$.

For each vertex $v_{i} \in \mathcal{S}^{*}$, let $\gamma_{i}(t)$ be the unique geodesic ray from $s$ to $-s$ through $v_{i}$ such that $\gamma_{i}(0)=s, \gamma_{i}(1)=v_{i}$, and $\gamma_{i}^{\prime}(t)=\gamma_{j}^{\prime}(t)$ for all $i, j$. Let $\mathbb{S}^{n-1}$ be the equator $\mathbb{S}^{n} \cap\left(\mathbb{E}^{n} \times\{0\}\right)$ and $\hat{t}=\min \left\{t: \gamma_{j}(t) \in \mathbb{S}^{n-1}\right.$ for some $\left.j\right\}$, that is, the first time that some $\gamma_{j}(t)$ reaches the equator $\mathbb{S}^{n-1}$. Denote $u_{i}=\gamma_{i}(\hat{t})$ for all $i$. Note that by the construction, the vertices $u_{1}, \ldots, u_{m} \in \mathbb{S}^{n-1}$ and $u_{m+1}, \ldots, u_{n+1}$ lie in the open hemi-sphere $\mathbb{S}^{n} \cap\left(\mathbb{E}^{n} \times[-1,0)\right)$ of $\mathbb{S}^{n}$.

As a corollary of Lemma 4, we have,

Corollary 5. For $t \in(1, \hat{t}], d_{\mathbb{S}^{n}}\left(\gamma_{i}(t), \gamma_{j}(t)\right)>d_{\mathbb{S}^{n}}\left(v_{i}, v_{j}\right)$ for $i \neq j$. In particular,

$$
d_{\mathbb{S}^{n}}\left(u_{i}, u_{j}\right)>d_{\mathbb{S}^{n}}\left(v_{i}, v_{j}\right)=\pi-\sigma_{i j} .
$$

Proposition 6.

1. The set $\left\{v_{1}, \ldots, v_{m}\right\}$ does not lie in any open hemi-sphere in $\partial B_{s}$. 
2. The vectors $u_{1}, \ldots, u_{n+1}$ do not lie in any open hemi-sphere in $\mathbb{S}^{n}$. In particular, the vectors $u_{1}, \ldots, u_{n+1}$ are linearly dependent.

Proof. To prove the first statement, we suppose otherwise. Then there is a unit vector $w$ so that the inner product $\left(w, v_{i}\right)>0$ for $i=1, \ldots, m$. Now move the center $s$ along the great circle $w_{t}=\frac{(1-t) s+t w}{\|(1-t) s+t w\|}$ where $t \in(0,1)$. An easy calculation using $\left(w, v_{i}\right)>0$ for $i=1, \ldots, m$ shows that $d_{\mathbb{S}^{n}}\left(w_{t}, v_{j}\right)<r=\operatorname{radius}\left(B_{s}\right)$ for all $j=1, \ldots, n+1$ and for small $t>0$. This contradicts the assumption that $B_{s}$ has the smallest radius.

To see the second statement, suppose otherwise that $u_{1}, \ldots, u_{n+1}$ lie in an open hemi-sphere in $S^{n}$. Then the open hemi-sphere intersects $\mathbb{S}^{n-1}$ in an open hemisphere. Since $u_{1}, \ldots, u_{m}$ are in $\mathbb{S}^{n-1}$, it follows that $u_{1}, \ldots, u_{m}$ also lie in an open hemi-sphere in $\mathbb{S}^{n-1}$. The spherical radial rays from $s$ determine a radial projection from $\partial B_{s}$ to $\mathbb{S}^{n-1}$ such that each $v_{i}$ is projected correspondingly to $u_{i}$ for $i=1, \ldots, n+1$. Furthermore, the radial projection sends hemi-spheres to hemispheres. Thus, $v_{1}, \ldots, v_{m}$ also lie in an open half $(n-1)$-ball in $\partial B_{s}$. This contradicts the first statement.

Since any $n+1$ independent unit vectors in $\mathbb{S}^{n}$ lie in an open hemi-sphere, the last statement follows.

First proof of M1. By proposition 6, there is an $n$-dimensional linear subspace $P$ of $\mathbb{E}^{n+1}$ containing the set $\left\{u_{1}, \ldots, u_{n+1}\right\}$. Then these points lie in the $(n-1)$-sphere denoted by $\mathbb{S}_{1}^{n-1}=\mathbb{S}^{n} \cap P$. Again by Proposition $6,\left\{u_{1}, \ldots, u_{n+1}\right\}$ does not lie in any open hemi-sphere of $\mathbb{S}_{1}^{n-1}$. Now, we may make use of the following lemma to finish.

Lemma 7. [GL, Lemma 5] Let $\left\{u_{1}, \ldots, u_{n+1}\right\} \subset \mathbb{S}^{n-1}$ which does not lie in any open hemi-sphere of $\mathbb{S}^{n-1}$. For every $\varepsilon>0$, there is a set $\left\{w_{1}, \ldots, w_{n+1}\right\} \subset \mathbb{S}^{n-1}$ such that it does not lie in any hemi-sphere of $\mathbb{S}^{n-1}$ and $d_{\mathbb{S}^{n}}\left(w_{i}, u_{i}\right)<\varepsilon$ for all $i$.

By this lemma, for $\varepsilon=\frac{1}{2} \min \left\{d\left(u_{i}, u_{j}\right)-d\left(v_{i}, v_{j}\right): i \neq j\right\}$, we find the points $w_{1}, \ldots, w_{n+1} \in \mathbb{S}_{1}^{n-1}$ such that $d\left(w_{i}, u_{i}\right)<\varepsilon$ for all $i$ and $\left\{w_{1}, \ldots, w_{n+1}\right\}$ does not lie in any hemi-sphere in $\mathbb{S}_{1}^{n-1}$. By the choice of $\varepsilon$, we have $d\left(w_{i}, w_{j}\right)>d\left(v_{i}, v_{j}\right)$ for all $i \neq j$. By Lemma $3, \mathcal{E}=\left\{x \in P:\left\langle\left(x-w_{i}\right), w_{i}\right\rangle \leq 0\right\}$ is an Euclidean $n$-simplex whose dihedral angles are given by $\pi-d\left(w_{i}, w_{j}\right)<\pi-d\left(v_{i}, v_{i}\right)=\sigma_{i j}$.

This completes the proof of Theorem M1.

The position of the center $s$ of $B_{s}$ in the dual simplex $\mathcal{S}^{*}$ has interesting geometric implication to the construction of the required $\mathbb{E}^{n}$-simplex. In fact, due to the convexity, we always have $s \in \mathcal{S}^{*}$. The crucial information is given by whether and how $s$ lies in $\partial B_{s}$. The following two propositions describe the geometric configuration about the vertices $v_{i}$ 's, the corresponding $u_{i}$ 's and the center $s$.

Proposition 8. The followings are true when $s$ lies in the interior of $\mathcal{S}^{*}$.

1. $m=n+1$.

2. $B_{s}$ is the $n$-ball circumscribing $\mathcal{S}^{*}$, i.e., $v_{i} \in \partial B_{s}$ for all $i=1, \ldots, n+1$.

3. The set $\left\{u_{1}, \ldots, u_{n+1}\right\}$ does not lie in any hemi-sphere of $\mathbb{S}^{n-1}$.

Proof. The first two results follow directly from a special case $(\ell=n+1)$ of Lemma 10 below. To get the last statement, one only needs to follow the argument of Proposition 6.

Note that the converse is not true, i.e., even if $m=n+1$, one may have $s \in \partial \mathcal{S}^{*}$. 
Proposition 9. The followings are true when $s$ lies on the boundary of $\mathcal{S}^{*}$.

1. There is an integer $\ell \leq n$ with $2 \leq \ell \leq m \leq n+1$ such that $\ell-1$ is the minimum dimension of a face of $\mathcal{S}^{*}$ which contains $s$.

2. $s$ lies in the interior of the face of $\mathcal{S}^{*}$ determined by $v_{1}, \ldots, v_{\ell}$.

3. $s$ is the center of a geodesic $(\ell-1)$-sphere circumscribing $\left\{u_{1}, \ldots, u_{\ell}\right\}$.

4. $\left\{u_{1}, \ldots, u_{\ell}\right\}$ is the vertex set of a compact Euclidean $(\ell-1)$-simplex with the origin as circumcenter.

5. $\left\{u_{1}, \ldots, u_{\ell}\right\} \subset \mathbb{E}^{n} \times\{0\}$ is of rank $(\ell-1)$ and $\left\{u_{\ell+1}, \ldots, u_{n+1}\right\}$ is linearly independent. In addition, $\left\{u_{1}, \ldots, u_{\ell}, \ldots, u_{n+1}\right\}$ is of rank $n$.

The following lemma is useful in the proofs of both propositions.

LEMma 10. If the center $s$ of $B_{s}$ lies in the interior of the $(\ell-1)$-face $\left(v_{1}, \ldots, v_{\ell}\right)$ for some $\ell \leq n+1$, then $B_{s} \cap \mathfrak{S}$ is the $(\ell-1)$-ball circumscribing $\left(v_{1}, \ldots, v_{\ell}\right)$, where $\mathfrak{S}$ is the totally geodesic $(\ell-1)$-sphere containing $\left\{v_{1}, \ldots, v_{\ell}\right\}$.

Proof. It is sufficient to show that $v_{1}, \ldots, v_{\ell} \in \partial B_{s}$. If $\partial B_{s} \cap \mathfrak{S}=\mathfrak{S}$, then we are done. If $\partial B_{s} \cap \mathfrak{S} \neq \mathfrak{S}$, suppose some of $v_{i}$ 's lie in the interior of $B_{s}$ in $\mathbb{S}^{n}$. Without loss of generality, let $k<\ell$ and $\left\{v_{1}, \ldots, v_{k}\right\} \subset \partial B_{s}$ while $v_{k+1}, \ldots, v_{\ell} \in B_{s}$. Since $s$ lies in the interior of $\left(v_{1}, \ldots, v_{\ell}\right)$ and $\operatorname{radius}\left(B_{s}\right)<\pi / 2$, it does not lie in the geodesic $(k-1)$-sphere spanned by $v_{1}, \ldots, v_{k}$. By the proof of Proposition 6 , we may perturb $s$ to $s^{\prime}$ and have a ball of smaller radius.

Proof. [Proof of Proposition 9] Let $\ell-1$ be the lowest dimension of a face of $\left(v_{1}, \ldots, v_{n+1}\right)$ that contains the center $s$. Obviously, $\ell \geq 2$ and by the minimality of $\ell, s$ lies in the interior of the face. Without loss of generality, assume this face has vertices $\left\{v_{1}, \ldots, v_{\ell}\right\}$ and it determines a totally geodesic $(\ell-1)$-sphere $\mathfrak{S}$. By Lemma $10, B_{s} \cap \mathfrak{S}$ is the $(\ell-1)$-ball circumscribing $\left\{v_{1}, \ldots, v_{\ell}\right\}$. Thus, $\ell \leq m$. Using the same argument as in Proposition 6, we can see that $\left\{u_{1}, \ldots, u_{\ell}\right\}$ does not lie in any open half $(\ell-1)$-ball of $\mathfrak{S} \cap \mathbb{S}^{n-1}$. Thus, it determines a compact Euclidean $(\ell-1)$-simplex. The last statement now follows from the nondegeneracy of $\mathcal{S}^{*}$ and a dimension count. $\square$

Based on Propositions 8 and 9, we are now giving a more explicit alternative proof for Theorem M1.

Second proof of M1. First, let us consider the case that $s \in\left(\mathcal{S}^{*}\right)^{\circ}$. By Proposition $8, B_{s}$ is the circumscribe $n$-ball of $\mathcal{S}^{*}$ and for all $i, j$, we have

$$
d_{\mathbb{S}^{n}}\left(u_{i}, u_{j}\right)>d_{\mathbb{S}^{n}}\left(v_{i}, v_{j}\right)=\pi-\sigma_{i j} .
$$

Moreover,

$$
\left\{u_{1}, \ldots, u_{n+1}\right\} \subset \mathbb{S}^{n-1} \subset \mathbb{E}^{n} \times\{0\} \subset \mathbb{E}^{n+1} ;
$$

but it does not lie in any closed half $(n-1)$-ball of $\mathbb{S}^{n-1}$.

Let $\mathcal{E}$ be the subset of $\mathbb{E}^{n} \times\{0\}$ bounded by the codimension- 1 hyperplanes tangent to $\mathbb{S}^{n-1}$ at the $u_{i}$ 's. Since the $u_{i}$ 's do not lie in any closed half-space, these tangent hyperplanes bound a compact Euclidean $n$-simplex $\mathcal{E}$ in $\mathbb{E}^{n} \times\{0\}$ with dihedral angles $\xi_{i j}=\pi-d_{\mathbb{S}^{n}}\left(u_{i}, u_{j}\right)<\sigma_{i j}$. So, $\mathcal{E}$ is the required Euclidean $n$-simplex.

In the case that $s \in \partial \mathcal{S}^{*}$, by Proposition 9, statement (4), there exists $a_{i}>0$, $i=1, \ldots, \ell$, such that $\sum_{i=1}^{\ell} a_{i} u_{i}=0$. Take arbitrarily small $\delta>0$ and let

$$
w_{i}= \begin{cases}u_{i}-\delta\left(u_{\ell+1}+\cdots+u_{n+1}\right) & i=1, \ldots, \ell, \\ u_{i} & i=\ell+1, \ldots, n+1 .\end{cases}
$$


One may choose $b_{i}>0$ as follows,

$$
b_{i}= \begin{cases}a_{i} /\left(\sum_{q=1}^{\ell} a_{q}\right) & i=1, \ldots, \ell \\ \delta & i=\ell+1, \ldots, n+1 .\end{cases}
$$

Then,

$$
\sum_{i=1}^{n+1} b_{i} w_{i}=\sum_{i=1}^{\ell} \frac{a_{i}}{\sum_{q=1}^{\ell} a_{q}} u_{i}-\sum_{i=1}^{\ell} \frac{a_{i} \delta}{\sum_{q=1}^{\ell} a_{q}} \sum_{j=\ell+1}^{n+1} u_{j}+\delta \sum_{i=\ell+1}^{n+1} u_{i}=0 .
$$

Next, we will prove that one may choose $\delta>0$ such that any subset of $n$ vectors among $\left\{w_{1}, \ldots, \ldots, w_{n+1}\right\}$ is linearly independent. We will consider the subset $\left\{w_{1}, \ldots, \psi_{q}, \ldots, w_{n+1}\right\}$ in the cases that $q \leq \ell$ or $q \geq \ell+1$.

Let $q \leq \ell$ and $\sum_{q \neq i=1}^{n+1} x_{i} w_{i}=0$. Substituting the expressions of $w_{i}$ 's, we have

$$
\sum_{\substack{i=1 \\ i \neq q}}^{\ell} x_{i} u_{i}+\sum_{i=\ell+1}^{n+1}\left(x_{i}-\delta \sum_{\substack{j=1 \\ j \neq q}}^{\ell} x_{j}\right) u_{i}=0
$$

Observe that if $q \leq \ell$, by (5) of Proposition $9,\left\{u_{1}, \ldots, k_{q}, \ldots, u_{n+1}\right\}$ is linearly independent. The above equation implies that $x_{i}=0$ for all $i \neq q$.

In the case that $q \geq \ell+1$ and $\sum_{q \neq i=1}^{n+1} x_{i} w_{i}=0$ for some $x_{i}$ 's and a certain $\delta>0$. We claim that only one specific $\delta$ may have nontrivial $x_{i}$ 's. By substituting the expressions of $w_{i}$ 's, we have

$$
\sum_{i=1}^{\ell} x_{i} u_{i}-\delta\left(\sum_{j=1}^{\ell} x_{j}\right) u_{q}+\sum_{\substack{i=\ell+1 \\ i \neq q}}^{n+1}\left(x_{i}-\delta \sum_{j=1}^{\ell} x_{j}\right) u_{i}=0 .
$$

Since $\left\{u_{1}, \ldots, u_{n+1}\right\}$ has rank $n$, the above equation has a one-dimensional space for the coefficients. If there are $\delta_{1}, \delta_{2}>0$ and corresponding $x_{i}^{(1)}, x_{i}^{(2)}$ which satisfy the above equation $(\star)$, one can conclude that

$$
\delta_{1}=\delta_{2} \quad \text { or } \quad \sum_{i=1}^{\ell} x_{i}^{(1)}=\sum_{i=1}^{\ell} x_{i}^{(2)}=0 .
$$

We will rule out the second alternative. Suppose there is a non-trivial set of $x_{i}$ 's with $\sum_{i=1}^{\ell} x_{i}=0$ such that $(\star)$ holds. Then, equation $(\star)$ becomes

$$
\sum_{i=1}^{\ell} x_{i} u_{i}+\sum_{\substack{i=\ell+1 \\ i \neq q}}^{n+1} x_{i} u_{i}=0
$$

By (5) of Proposition 9, the vectors $\left\{u_{i}\right\}_{i=1}^{\ell}$ and $\left\{u_{i}\right\}_{i=\ell+1}^{n+1}$ span direct summands. Thus, we must have simultaneously

$$
\sum_{i=1}^{\ell} x_{i} u_{i}=0, \quad \sum_{\substack{i=\ell+1 \\ i \neq q}}^{n+1} x_{i} u_{i}=0
$$


However, $\sum_{i=1}^{\ell} x_{i} u_{i}=0$ together with $\sum_{i=1}^{\ell} x_{i}=0$ contradict that $u_{1}, \ldots, u_{\ell}$ form a compact Euclidean simplex. Consequently, one must have $\delta_{1}=\delta_{2}$.

Thus, by [GL, Lemma 4], there is sufficiently small $\delta>0$ such that the vertices $w_{i}, i=1, \ldots, n+1$ span an $n$-dimensional space $L \subset \mathbb{R}^{n+1}$ and they define a compact Euclidean $n$-simplex in $L$. Furthermore, $\left\|w_{i}-u_{i}\right\|$ can be made arbitrarily small. Let $\mathcal{E}$ be the Euclidean $n$-simplex in $L$ dual to $w_{i}$ 's. In other words, if $w_{i}$ 's are normalized, $\mathcal{E}$ is bounded by the tangent hyperplanes to $\mathbb{S}^{n} \cap L$ at $w_{i}$. Its dihedral angles $\xi_{i j}$ satisfy that $\left|\xi_{i j}-\left(\pi-\ell_{i j}\right)\right|<\varepsilon$ for arbitrarily small $\varepsilon>0$. Hence,

$$
\xi_{i j}<\pi-\ell_{i j}+\varepsilon<\pi-d_{\mathbb{S}^{n}}\left(v_{i}, v_{j}\right)=\sigma_{i j} .
$$

This completes the proof of the theorem.

\section{REFERENCES}

[AVS] D. V. Alekseevskij, E. B. Vinberg, And A. S. Solodovnikov, Geometry of spaces of constant curvature. Geometry II, pp. 1-138. Encyclopaedia Math. Sci., 29, Springer, Berlin, 1993.

[Dia] R. Díaz, A characterization of Gram matrices of polytopes, Discrete Comput. Geom., 21 (1999), pp. 581-601.

[GL] R. Guo And F. Luo, On a Conjecture of Milnor about Volume of Simplexes, arXiv:math. GT/0510666.

[LL] H. J. Lindsey and O. L. Lossers, A Similarity Criterion, American Mathematical Monthly, 105 (1998), p. 671.

[Luo1] F. Luo, On a problem of Fenchel, Geom. Ded., 64 (1997), pp. 277-282.

[Luo2] F. Luo, A Characterization of Spherical Cone Metrics on Surfaces, arXiv:math. GT/0408112.

[Luo3] F. Luo, Continuity of the Volume of Simplices in Classical Geometry, Communications in Contemporary Mathematics, to appear.

[Mil] J. Milnor, The Schläfli differential equality, Collected Papers., vol. 1, Houston: Publish or Perish, 1994.

[RH] I. Rivin AND C. D. Hodgson, A characterization of compact convex polyhedra in hyperbolic 3-space, Invent. Math., 111 (1993), pp. 77-111.

[Riv1] I. RIVIN, On geometry of convex ideal polyhedra in hyperbolic 3-space, Topology, 32 (1993), pp. 87-92.

[Riv2] I. Rivin, Problem 10462, American Mathematical Monthly, 102 (1995), p. 554.

[Riv3] I. Rivin, A characterization of ideal polyhedra in hyperbolic 3-space, Ann. of Math., 143 (1996), pp. 51-70. 